\title{
Dual specificity phosphatase 6 suppresses the growth and metastasis of prostate cancer cells
}

\author{
XIAOLEI ZHAI, QIANHE HAN, ZHONGJIE SHAN, XIAOWEI QU, LIANG GUO and YUDONG ZHOU \\ Department of Urology, People's Hospital of Zhengzhou, Zhengzhou, Henan 450003, P.R. China
}

Received October 14, 2013; Accepted June 5, 2014

DOI: $10.3892 / \mathrm{mmr} .2014 .2575$

\begin{abstract}
Dual specificity phosphatase 6 (DUSP6) is expressed at low levels in numerous types of human cancer. The loss of DUSP6 plays a pivotal role in tumor progression; however, the role of DUSP6 in prostate cancer remains unclear. In this study, in vitro invasion assays and in vivo metastasis experiments were used to investigate the effects of DUSP6 on prostate cancer cell invasion and metastasis. Furthermore, in vitro growth and soft agar assays and in vivo growth experiments were performed to determine the function of DUSP6 in cell proliferation. The results showed that the overexpression of DUSP6 suppressed the invasion and growth of DU-145 human prostate cancer cells, whereas knockdown of DUSP6 promoted the invasion and proliferation of LNCap human prostate adenocarcinoma cells. Further experiments demonstrated that the overexpression of DUSP6 inhibited the proliferation and liver metastasis of DU-145 cells in mice. In addition, DUSP6 downregulated the expression of matrix metallopeptidase 3 and interleukin 8 in prostate cancer cells. Taken together, these findings indicate that DUSP6 may act as a negative mediator in the regulation of prostate cancer cell growth and metastasis.
\end{abstract}

\section{Introduction}

Prostate cancer is one of the most common cancers in males and has become a leading cause of cancer-related mortality worldwide (1). Tumor invasion, metastasis and uncontrolled growth are hallmarks of this type of mortality (2). Numerous molecules expressed by cancer cells can be either up- or downregulated during the disease progression, and this is believed to play a pivotal role in cancer cell proliferation and metastasis (3). Thus, investigations into these molecules may

Correspondence to: Professor Yudong Zhou, Department of Urology, People's Hospital of Zhengzhou, 33 Huang He Road, Zhengzhou, Henan 450003, P.R. China

E-mail: ydzhoumed@163.com

Key words: dual specificity phosphatase 6, metastasis, growth, prostate cancer, matrix metallopeptidase 3 , interleukin 8 provide an enhanced understanding of tumor occurrence and progression.

The dual specificity phosphatases, also termed mitogen-activated protein kinase (MAPK) phosphatases, consist of ten catalytically active proteins and act as negative regulators of MAPK activity in mammalian cells (4).Dual specificity phosphatase 6 (DUSP6) is a member of the DUSP gene family and typically shows reduced expression in numerous types of cancer, including lung (5) and pancreatic (6) cancer. Previous study demonstrated that DUSP6 is underexpressed in ovarian cancer and a low level of DUSP6 is inversely correlated with high levels of phospho-extracellular signal-regulated kinase (ERK)1/2 (7). The loss of DUSP6 contributes to the inhibition of apoptosis in ovarian cancer (7) and is associated with epithelial-mesenchymal transition (EMT) and cell invasion in esophageal squamous cell and nasopharyngeal carcinomas (8). By contrast, other studies have found that increased expression levels of DUSP6 can stimulate tumorigenesis in melanoma and breast cancer $(9,10)$ and overexpression of DUSP6 is closely associated with the invasion of papillary thyroid cancer (11), indicating that DUSP6 exerts contrasting effects on different cancer types. The role of DUSP6 in prostate cancer remains poorly understood; therefore, the present study aimed to examine the involvement of DUSP6 in prostate cancer cell growth and metastasis and to elucidate the underlying molecular mechanisms.

\section{Materials and methods}

Antibodies and cell lines. Antibodies against DUSP6, matrix metallopeptidase 3 (MMP-3) and $\beta$-actin were purchased from Santa Cruz Biotechnology, Inc. (Santa Cruz, CA, USA). The cell lines (RWPE-1, PC-3, LNCap and DU-145) were purchased from the cell bank of the Chinese Academy of Sciences (Shanghai, China). RWPE-1 cells (a normal prostate cell line) were maintained in keratinocyte serum-free medium containing $10 \%$ fetal bovine serum (FBS). Prostate cancer cells (PC-3, LNCap and DU-145) were cultured in RPMI-1640 medium supplemented with $10 \%$ FBS. All cells were cultured at $37^{\circ} \mathrm{C}$ within a humidified atmosphere containing $5 \% \mathrm{CO}_{2}$.

Generation of stable cells. To generate DU-145 cells overexpressing DUSP6, the cells were transfected with a pcDNA 3.1-DUSP6 vector that was designed and purchased from Sangon Biotech (Shanghai, China). DU-145 cells transfected 
with an empty pcDNA 3.1 vector were used as a negative control. To silence the expression of DUSP6 in LNCap cells, the cells were transfected with DUSP6 short hairpin RNA (shRNA) vector, purchased from Shanghai GenePharma Co., Ltd. (Shanghai, China). Cells transfected with a scrambled-sequence shRNA vector were used as a negative control. G418 (Gibco-BRL, Grand Island, NY, USA) was used to isolate the stable cells.

Quantitative polymerase chain reaction ( $q P C R)$. Total RNA was isolated using TRIzol ${ }^{\circledR}$ reagent (Invitrogen Life Technologies, Carlsbad, CA, USA). cDNA was then obtained through reverse transcription using a cDNA Synthesis kit (Promega Corp., Madison, WI, USA). qPCR was performed using the primers listed in Table I. The qPCR cycling conditions comprised $10 \mathrm{~min}$ at $95^{\circ} \mathrm{C}$, and 40 cycles of $15 \mathrm{sec}$ at $95^{\circ} \mathrm{C}$ and $1 \mathrm{~min}$ at $60^{\circ} \mathrm{C}$. The expression levels of the examined genes were normalized to $\beta$-actin and the $2^{-\Delta \Delta \mathrm{Ct}}$ method was used to determine relative quantification (12).

Cell lysis and western blotting. Cells were lysed in radioimmunoprecipitation assay buffer with protease inhibitors (Roche, Mannheim, Germany). A bicinchoninic acid protein assay (Applygen Technologies, Inc., Beijing, China) was then used to determine the protein concentration of each sample. Equal amounts of protein were subsequently separated by SDS-PAGE and the gel was transferred to a polyvinylidene fluoride membrane (Bio-Rad, Hercules, CA, USA). The membrane was blocked with 5\% milk and probed for DUSP6 (1:500), MMP-3 (1:500) and $\beta$-actin $(1: 1,000)$. The membrane was then further incubated with the secondary antibody (Sigma, St. Louis, MO, USA) for $1 \mathrm{~h}$ at room temperature. The immunoreactive bands were exposed to a film and quantified by Quantity One software (Bio-Rad).

ELISA. To detect the expression level of interleukin 8 (IL-8) secreted by the prostate cancer cells, the cell supernatant was collected and an IL-8 ELISA kit (Boster Biological Technology Co., Ltd., Wuhan, China) was used to measure the protein level of IL-8. Samples were assayed in duplicate. The optical density values were read at $450 \mathrm{~nm}$ using a microtiter plate reader within 30 min of performing the reaction, and readings were compared with a standard curve. The concentration of IL- 8 protein was normalized to the total protein levels.

In vitro invasion and migration assays. A 24-well Transwell plate (Costar, Corning, NY, USA) was used for the invasion and migration assays. For the invasion assay, cells were harvested and resuspended in RPMI-1640 medium supplemented with $0.1 \%$ bovine serum albumin. The upper chambers were coated with Matrigel ${ }^{\mathrm{TM}}$ (BD Biosciences, Franklin Lakes, NJ, USA) and 100,000 cells were placed in the upper chambers. The lower chambers were filled with $600 \mu 1$ RPMI-1640 medium supplemented with 20\% FBS, and cells were allowed to invade for $24 \mathrm{~h}$. The cells on the lower surface of the membranes were fixed with $4 \%$ formaldehyde and stained with crystal violet. The number of invaded cells in seven fields was observed and counted under a microscope at a magnification of $\times 200$.
Table I. Quantitative polymerase chain reaction primers.

\begin{tabular}{|c|c|c|}
\hline Gene & Primer sequence $\left(5^{\prime}-3^{\prime}\right)$ & $\begin{array}{l}\text { Length } \\
\text { (bp) }\end{array}$ \\
\hline DUSP6 & $\begin{array}{l}\text { F: CCTGAGGCCATTTCTTTCATAGA } \\
\text { R: GTCACAGTGACTGAGCGGCTAAT }\end{array}$ & 115 \\
\hline MMP-3 & $\begin{array}{l}\text { F: CTGGACTCCGACACTCTGGA } \\
\text { R: CAGGAAAGGTTCTGAAGTGACC }\end{array}$ & 79 \\
\hline IL-8 & $\begin{array}{l}\text { F: ACTGAGAGTGATTGAGAGTGGAC } \\
\text { R: AACCCTCTGCACCCAGTTTTC }\end{array}$ & 112 \\
\hline$\beta$-actin & $\begin{array}{l}\text { F: GGATGCAGAAGGAGATCACTG } \\
\text { R: CGATCCACACGGAGTACTTG }\end{array}$ & 90 \\
\hline
\end{tabular}

F, forward; R, reverse; DUSP6, dual specificity phosphatase 6; MMP-3, matrix metallopeptidase 3; IL-8, interleukin 8.

For the migration assay, cells were resuspended at a density of $5.0 \times 10^{5}$ cells $/ \mathrm{ml}$. Cells in $100 \mu \mathrm{l}$ RPMI-1640 were placed on the top of the chambers. RPMI-1640 supplemented with $20 \%$ FBS was added to the lower chambers. The cells were incubated for $24 \mathrm{~h}$ at $37^{\circ} \mathrm{C}$. Following staining with crystal violet, the number of migrated cells was counted and determined from seven representative fields.

Growth and MTT assays. For the growth assay, cells were seeded in a 24-well culture plate at a density of $1.0 \times 10^{4}$ cells/well. The cells were harvested and counted using a hemocytometer stained with trypan blue on the day indicated. For the MTT assay, cells were seeded in a 96-well culture plate at an initial density of $0.5 \times 10^{3}$ cells/well. On the day indicated, MTT was added to the wells. Four hours later, the medium was removed and dimethyl sulfoxide was added. Following agitation for $15 \mathrm{~min}$ in the dark, the absorbance was measured at $490 \mathrm{~nm}$.

Soft agar assay. Dulbecco's Modified Eagle's Medium (DMEM) containing $0.6 \%$ agarose was added to a six-well culture plate on the bottom layer. Cells $\left(0.8 \times 10^{3}\right.$ cells/well $)$ mixed with DMEM containing $0.3 \%$ agarose were plated on top to form an upper layer. Cells were plated in triplicate and colony numbers were counted after 14 days.

In vivo growth and metastasis experiments. Male four-week-old BALB/c nude mice (Vital River Laboratories, Beijing, China) were maintained in pathogen-free conditions. All experimental protocols were approved by the Animal Care and Use Committee of Zhengzhou University (Zhengzhou, China). Each mouse was injected subcutaneously with $3.0 \times 10^{5}$ DU-145 Vector or DU-145 pcDUSP6 cells ( $n=6$ for each group). The length (L) and width (W) of the tumors in the mice were measured using calipers each week and the tumor volumes were estimated with the following formula: $0.52 \times \mathrm{L} \times \mathrm{W}^{2}$. After eight weeks the mice were sacrificed, the mean tumor weight was determined and the lungs and livers were dissected and fixed in $4 \%$ paraformaldehyde. The tissues were sectioned into slices and stained 

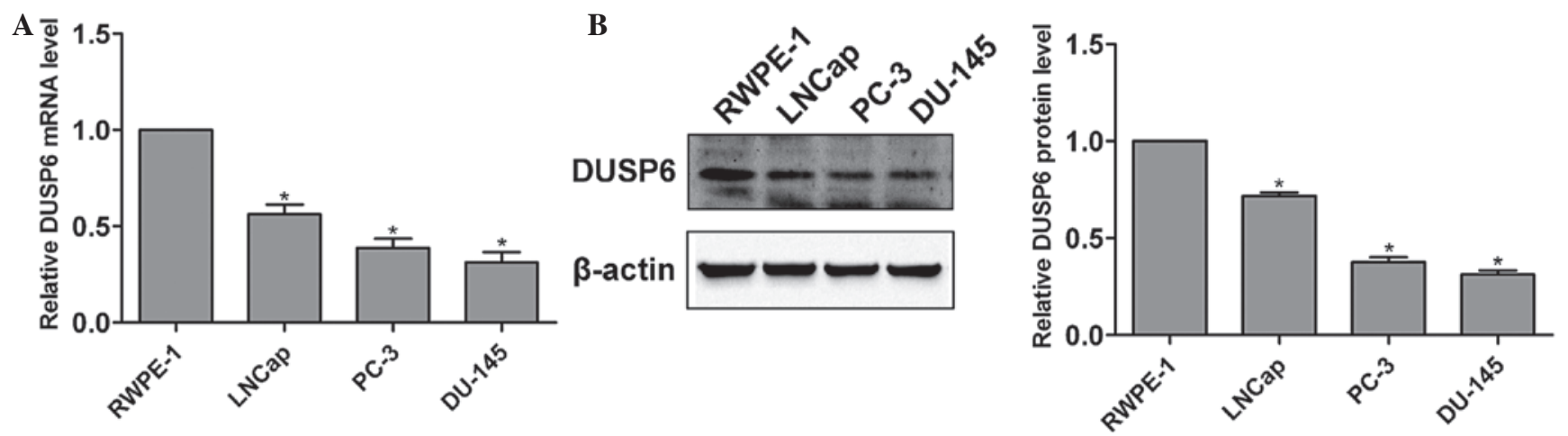

Figure 1. DUSP6 is expressed at low levels in prostate cancer cells. (A) The mRNA level of DUSP6 was detected using the quantitative polymerase chain reaction in RWPE-1, LNCap, PC-3 and DU-145 cells. (B) The protein level of DUSP6 was observed by western blotting. Three independent experiments were performed. The expression of DUSP6 in RWPE- 1 cells was defined as 1 and used as a control. Data are presented as the mean \pm standard deviation. $\mathrm{P}<0.05$ versus the RWPE-1 cells. DUSP6, dual specificity phosphatase 6 .

with hematoxylin and eosin. Each section was observed by microscopy.

Statistical analysis. Data are presented as the mean \pm standard deviation. The data were analyzed using SPSS 17.0 software (SPSS Inc., Chicago, IL, USA). The Student's t test was used to compare two means, whilst nonparametric analysis of variance was performed to compare multiple means. $\mathrm{P}<0.05$ was considered to indicate a statistically significant difference.

\section{Results}

DUSP6 expression is decreased in prostate cancer cells. To determine the expression of DUSP6, qPCR and western blotting were performed in a normal prostate cell line (RWPE-1) and three prostate cancer cell lines (LNCap, PC-3 and DU-145). The results showed that DUSP6 was significantly expressed in the RWPE-1 cells, whereas the expression of DUSP6 was low in the analyzed prostate cancer cells (Fig. 1A and B).

DUSP6 inhibits the invasion and migration of prostate cancer cells in vitro. To investigate the role of DUSP6 in prostate cancer cells, a cell clone stably overexpressing DUSP6 was isolated from DU-145 cells (pcDUSP6) and a cell clone transfected with an empty vector was used as a negative control (Vector). In the LNCap cells, a DUSP6 shRNA vector was used to stably silence the expression of DUSP6 (shDUSP6) and a scrambled-sequence shRNA vector was used as negative control (shVector). The cells stably expressing DUSP6 were analyzed by western blotting for protein expression levels (Fig. 2A) and were used for in vitro invasion and migration assays (Fig. 2B-D). The results showed that the overexpression of DUSP6 significantly suppressed the invasion and migration of DU-145 cells (Fig. 2B and D). Furthermore, as compared with the LNCap shVector cells, LNCap shDUSP6 cells showed increased invasion and migration abilities (Fig. 2C and E). These data indicated that DUSP6 can suppress the invasion and migration of prostate cancer cells in vitro.

DUSP6 negatively regulates the growth of prostate cancer cells in vitro. The role of DUSP6 in the growth of prostate cancer cells was next evaluated. Using growth and MTT assays, it was found that the overexpression of DUSP6 suppressed the growth of DU-145 cells, whereas DUSP6-knockdown promoted the growth of LNCap cells (Fig. 3A-D). In addition, a soft agar assay was performed to assess the anchorage-independent growth of prostate cancer cells. The results demonstrated that the overexpression of DUSP6 decreased the number of colonies of DU-145 cells, whereas knockdown of DUSP6 increased the number of colonies of LNCap cells (Fig. 3E and F). These data suggested that DUSP6 is involved in the negative regulation of prostate cancer cell growth in vitro.

DUSP6 suppresses prostate cancer cell growth and metastasis in vivo. To explore the functional role of DUSP6 in growth and metastasis in vivo, nude mice were injected subcutaneously with DU-145 Vector cells or DU-145 pcDUSP6 cells. The tumor volume was measured once per week. The data showed that DU-145 pcDUSP6 tumors exhibited a lower growth rate compared with DU-145 Vector tumors (Fig. 4A). After eight weeks, the mice were sacrificed and the mean tumor weight was assessed. The results showed that the mean tumor weight was $5.93 \pm 1.20 \mathrm{~g}$ for the DU-145 Vector tumors and $3.50 \pm 0.66 \mathrm{~g}$ for the DU-145 pcDUSP6 tumors $(\mathrm{P}<0.05)$ (Fig. 4B). These data indicated that the overexpression of DUSP6 can suppress the growth of prostate cancer cells in vivo. Furthermore, the liver metastasis rate of mice injected with DU-145 Vector cells was $100 \%$ (6/6), whilst that of mice injected with DU-145 pcDUSP6 cells was $16.7 \%$ (1/6) (Fig. 4C and D), indicating that DUSP6 can suppress the metastasis of prostate cancer cells in vivo.

DUSP6 reduces the expression of MMP-3 and IL-8 in prostate cancer cells. MMPs are crucial factors in the invasion and metastasis of prostate cancer cells (13). This study revealed that the overexpression of DUSP6 inhibited MMP-3 expression in DU-145 cells, whereas DUSP6-knockdown enhanced MMP-3 expression in LNCap cells (Fig. 5A). A consistent result was observed by examining the protein level of MMP-3 using western blotting (Fig. 5B). IL- 8 has been suggested to be associated with cell invasion and proliferation in prostate cancer $(14,15)$. Using qPCR and ELISA, it was found that the 
A

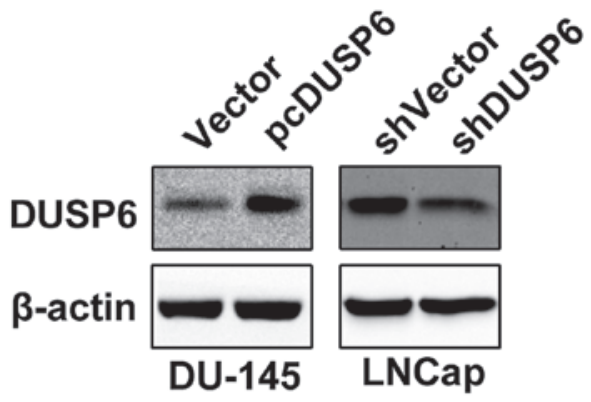

B
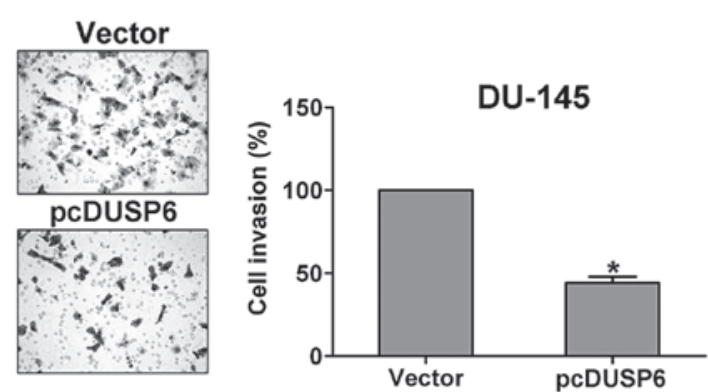

D
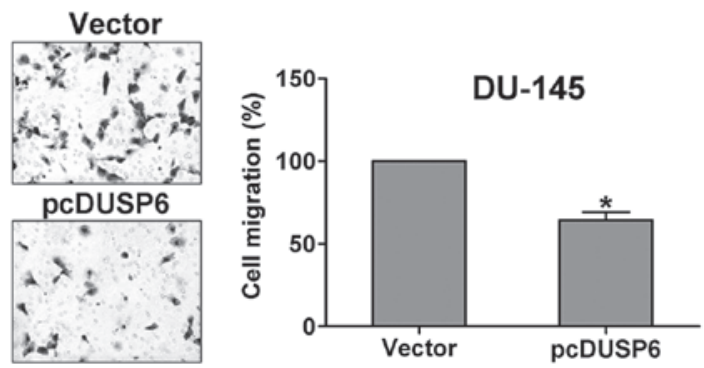

C

shVector
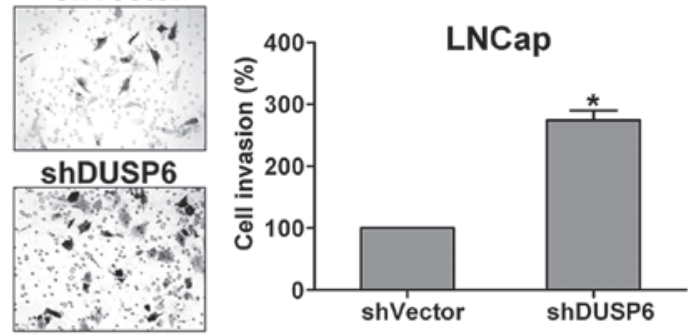

$\mathbf{E}$

shVector
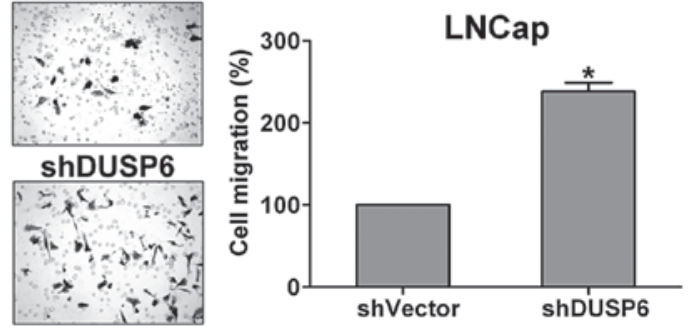

Figure 2. Effects of DUSP6 on the in vitro invasion and migration of prostate cancer cells. Cell clones stably transfected with a pcDNA-DUSP6 vector (pcDUSP6) or an empty pcDNA vector (Vector) were generated from DU-145 cells and cell clones stably transfected with a DUSP6 shRNA vector (shDUSP6) or a scramble shRNA vector (shVector) were isolated from LNCap cells. (A) The expression of DUSP6 in stably transfected cells was detected by western blotting. (B and C) Effects of DUSP6 overexpression on the invasion of (B) DU-145 and (C) LNCap cells. (D and E) Effects of DUSP6-knockdown on the migration of (D) DU-145 and (E) LNCap cells. Three independent experiments were performed. ${ }^{*} \mathrm{P}<0.05$ versus the controls. Data are presented as the mean \pm standard deviation. (Magnification, x200). DUSP6, dual specificity phosphatase 6; shRNA, short hairpin RNA.

A

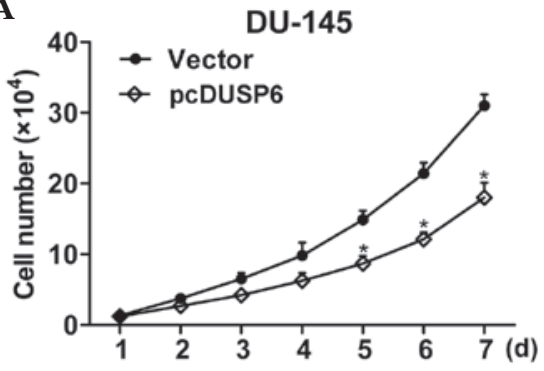

D

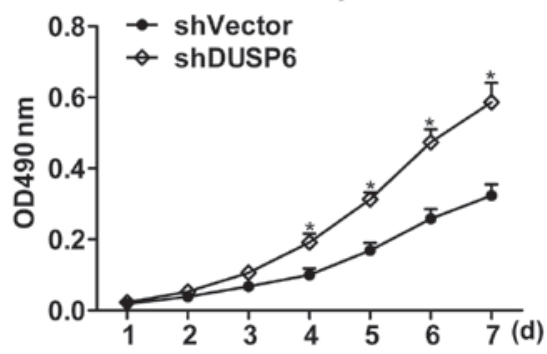

B

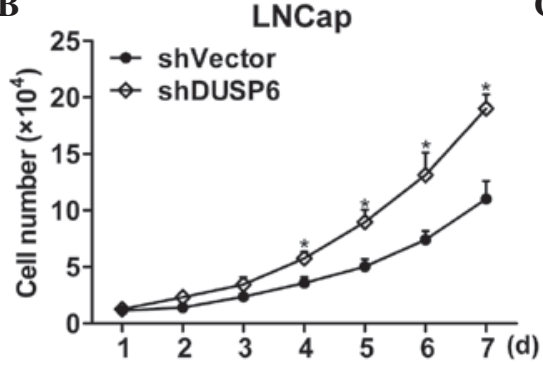

$\mathbf{E}$

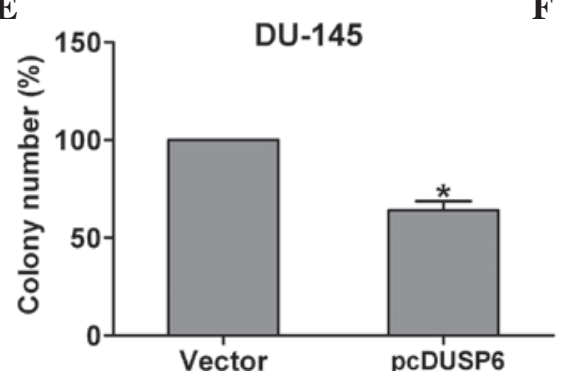

C

$\mathbf{F}$
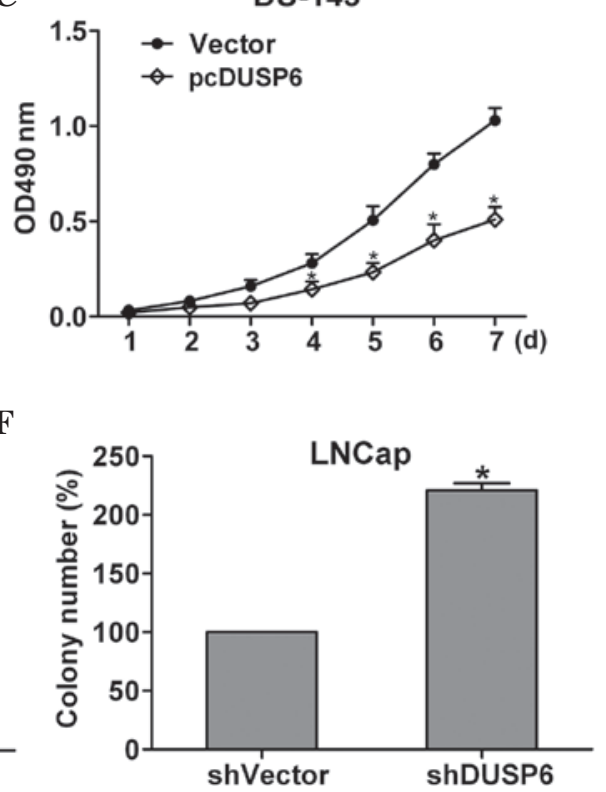

Figure 3. DUSP6 suppresses the growth and clonogenic ability of prostate cancer cells. (A and B) Cell growth, (C and D) MTT and (E and F) soft agar assays comparing (A, C and E) DU-145 Vector and DU-145 pcDUSP6 cells and (B, D and F) LNCap shVector and LNCap shDUSP6 cells. Three independent experiments were performed. "P $<0.05$ versus the controls. Data are presented as the mean \pm standard deviation. DUSP6, dual specificity phosphatase 6; shRNA, short hairpin RNA. 
A

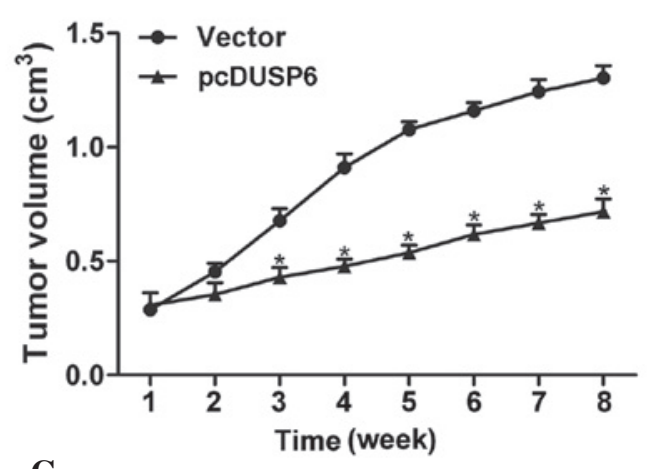

C

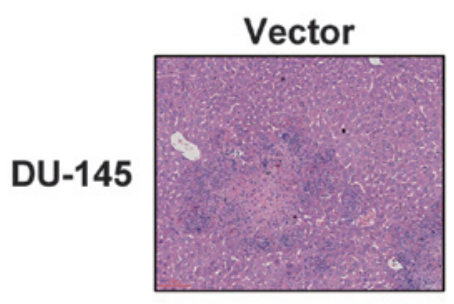

B
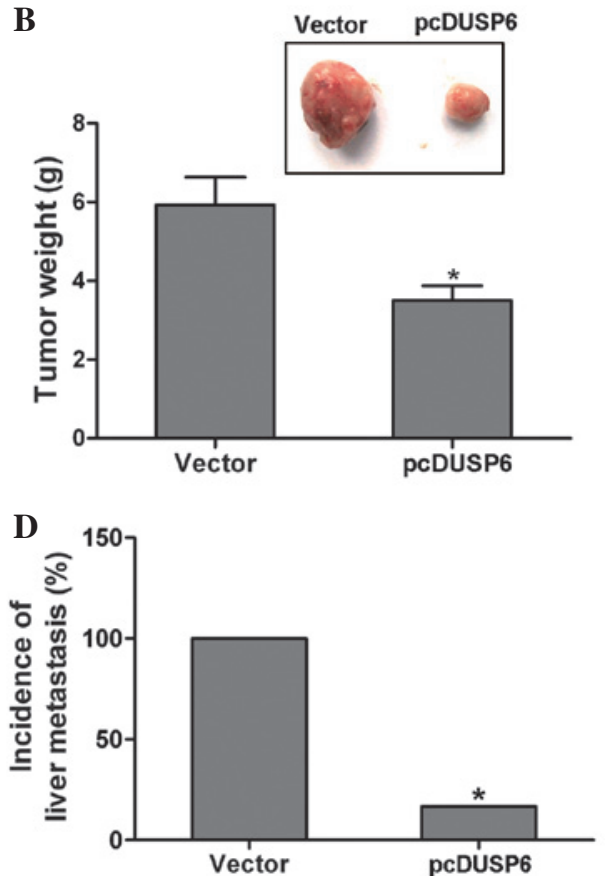

Figure 4. Overexpression of DUSP6 inhibits the growth and metastasis of prostate cancer cells in vivo. DU-145 Vector or DU-145 pcDUSP6 cells were injected subcutaneously in mice. (A) The tumor volume was measured once every week to assess the growth rate of DU-145 Vector and DU-145 pcDUSP6 cells in vivo. (B) Eight weeks later, the mice were sacrificed and the mean tumor weight was determined. (C) The micrometastasis in the liver section was observed under a microscope. Scale bars $=100 \mu \mathrm{m}$. (D) Incidence of live metastasis in mice that were injected with DU-145 Vector or DU-145 pcDUSP6 cells. "P<0.05 versus the Vector cells. Data are presented as the mean \pm standard deviation. (Magnification, x200). DUSP6, dual specificity phosphatase 6.
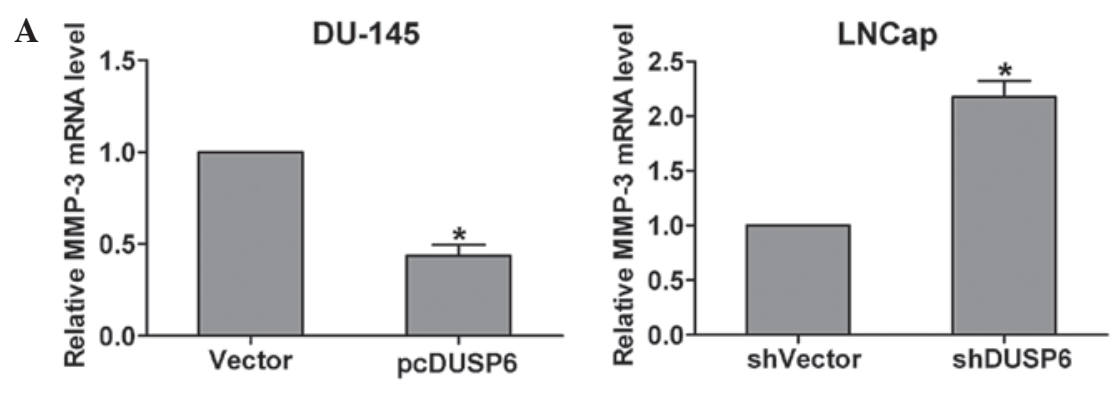

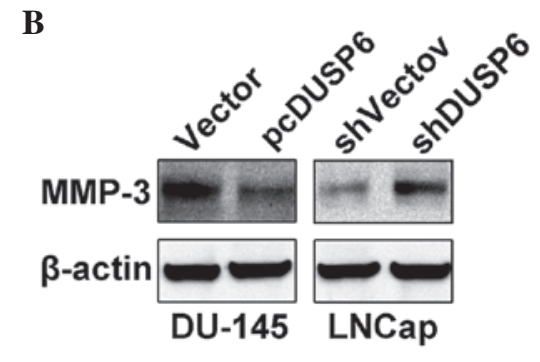

C

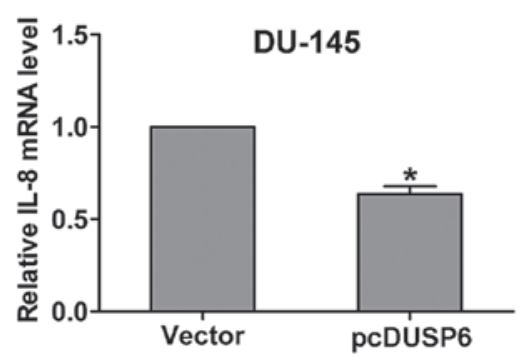

D

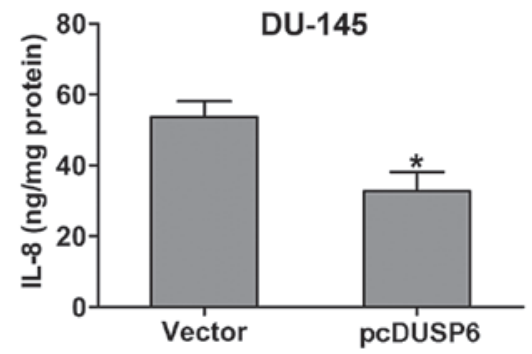

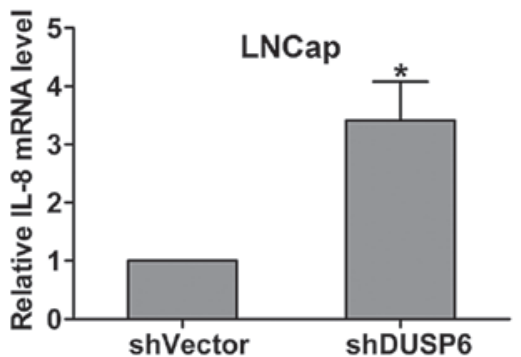

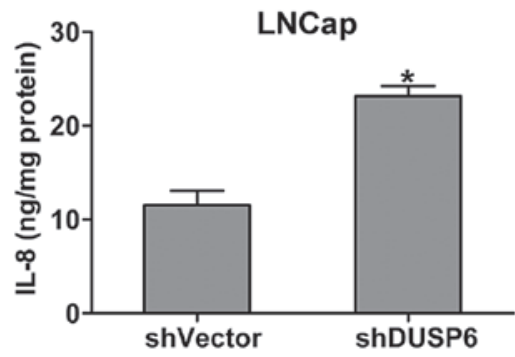

Figure 5. DUSP6 inhibits the expression of MMP-3 and IL-8 in prostate cancer cells. (A) qPCR and (B) western blotting were performed to examine the mRNA and protein levels of MMP-3, respectively. (C) qPCR and (D) ELISA were conducted to measure the mRNA and protein levels of IL-8, respectively. Three independent experiments were performed. " $\mathrm{P}<0.05$ versus the control. Data are presented as the mean \pm standard deviation DUSP6, dual specificity phosphatase 6; MMP-3, matrix metallopeptidase 3; IL-8, interleukin 8; qPCR, quantitative polymerase chain reaction. 
overexpression of DUSP6 suppressed the expression of IL-8 in DU-145 cells, whereas DUSP6-knockdown enhanced the level of IL-8 in LNCap cells (Fig. 5C and D). These results suggested that DUSP6 can downregulate the expression of MMP-3 and IL-8 in prostate cancer cells.

\section{Discussion}

There are 10 DUSPs expressed in mammalian cells (16). Amongst these, DUSP6 exhibits a specificity for the inactivation of ERKs (17). Studies have found that a loss of DUSP6 expression is correlated with tumor progression $(18,19)$. In this study, in vitro and in vivo data showed that the overexpression of DUSP6 suppressed the invasion and growth of DU-145 cells. By contrast, knockdown of DUSP6 enhanced the invasion and growth of LNCap cells. Further experiments showed that DUSP6 reduced the expression of MMP-3 and IL-8 in prostate cancer cells. Together, these findings strongly indicate that DUSP6 acts as a negative mediator in the regulation of prostate cancer cell growth and metastasis.

It has been previously reported that DUSP6 is expressed at low levels in lung and pancreatic cancers $(19,20)$, and loss of DUSP6 has additionally been shown to enhance the tumorigenicity and chemoresistance of ovarian cancer cells (7). In the present study, it was found that the expression of DUSP6 was decreased in prostate cancer cells, indicating that DUSP6 may be a candidate gene involved in the pathogenesis of prostate cancer. A previous study revealed that the upregulation of DUSP6 regulated p53-mediated apoptosis by decreasing the expression of B-cell lymphoma 2 in colorectal carcinoma cells (22). In lung adenocarcinomas, high DUSP6 expression has been shown to correlate with a lower histological grade and a lower Ki-67 index (23). Additionally, the overexpression of DUSP6 in pancreatic cancer cells has been shown to result in the suppression of cell growth through a reduction in phosphorylated ERK1/2 (24). The present study found that DUSP6 could negatively regulate the growth of prostate cancer cells. Consistent with the in vitro data, in vivo growth experiments demonstrated that the overexpression of DUSP6 suppressed the growth of prostate cancer cells. It has been reported that the loss of DUSP6 is associated with the invasion and progression of pancreatic cancer (6) and, in esophageal squamous cell and nasopharyngeal carcinoma, the overexpression of DUSP6 can suppress cell invasion and EMT (8). The results of the present demonstrated that DUSP6 could inhibit the in vitro invasion and migration of the DU-145 and LNCap cells. Furthermore, the overexpression of DUSP6 suppressed the metastasis of prostate cancer cells in vivo.

MMP-3 functions as a significant regulatory factor in the aggressiveness of tumors (25), and increased MMP-3 expression has been reported to be closely associated with tumor growth and metastasis $(26,27)$. Belonging to the CXC chemokine family, IL-8 is expressed at low levels in normal tissues but is highly expressed in tumor environments $(28,29)$. The crucial effect of IL-8 on prostate cancer cell proliferation and metastasis has also been demonstrated in numerous studies (30-32). It was found in the present study that DUSP6 could decrease the expression of both MMP-3 and IL-8 in prostate cancer cells, indicating that MMP-3 and IL-8 may be involved in the DUSP6-suppressed growth and metastasis of prostate cancer cells.
In conclusion, the present study has demonstrated that DUSP6 can inhibit prostate cancer cell growth, invasion and metastasis by downregulating the expression of MMP-3 and IL-8. Further studies are required to investigate the expression of DUSP6 in prostate tumor specimens.

\section{References}

1. Siegel R, Naishadham D and Jemal A: Cancer statistics, 2013. CA Cancer J Clin 63: 11-30, 2013.

2. Steeg PS: Tumor metastasis: mechanistic insights and clinical challenges. Nat Med 12: 895-904, 2006.

3. Martin GS: Cell signaling and cancer. Cancer Cell 4: 167-174, 2003.

4. Theodosiou A and Ashworth A: MAP kinase phosphatases. Genome Biol 3, 3009, 2002.

5. Okudela K, Yazawa T, Woo T, et al: Down-regulation of DUSP6 expression in lung cancer: its mechanism and potential role in carcinogenesis. Am J Pathol 175: 867-881, 2009.

6. Furukawa T, Fujisaki R, Yoshida Y, et al: Distinct progression pathways involving the dysfunction of DUSP6/MKP-3 in pancreatic intraepithelial neoplasia and intraductal papillary-mucinous neoplasms of the pancreas. Mod Pathol 18: 1034-1042, 2005.

7. Chan DW, Liu VW, Tsao GS, et al: Loss of MKP3 mediated by oxidative stress enhances tumorigenicity and chemoresistance of ovarian cancer cells. Carcinogenesis 29: 1742-1750, 2008.

8. Wong VC, Chen H, Ko JM, et al: Tumor suppressor dual-specificity phosphatase 6 (DUSP6) impairs cell invasion and epithelial-mesenchymal transition (EMT)-associated phenotype. Int J Cancer 130: 83-95, 2012.

9. Li W, Song L, Ritchie AM and Melton DW: Increased levels of DUSP6 phosphatase stimulate tumourigenesis in a molecularly distinct melanoma subtype. Pigment Cell Melanoma Res 25: 188-199, 2012.

10. Zhang YY, Wu JW and Wang ZX: Mitogen-activated protein kinase (MAPK) phosphatase 3-mediated cross-talk between MAPKs ERK2 and p38alpha. J Biol Chem 286: 16150-16162, 2011.

11. Lee JU, Huang S, Lee MH, et al: Dual specificity phosphatase 6 as a predictor of invasiveness in papillary thyroid cancer. Eur J Endocrinol 167: 93-101, 2012

12. Livak KJ and Schmittgen TD: Analysis of relative gene expression data using real-time quantitative PCR and the 2(-Delta Delta C(T)) Method. Methods 25: 402-408, 2001.

13. Yang JL, Lin JH, Weng SW, et al: Crude extract of Euphorbia formosana inhibits the migration and invasion of DU145 human prostate cancer cells: The role of matrix metalloproteinase-2/9 inhibition via the MAPK signaling pathway. Mol Med Rep 7: 1403-1408, 2013.

14. Lehrer S, Diamond EJ, Mamkine B, Stone NN and Stock RG: Serum interleukin-8 is elevated in men with prostate cancer and bone metastases. Technol Cancer Res Treat 3: 411, 2004.

15. Huang S, Pettaway CA, Uehara H, Bucana CD and Fidler IJ: Blockade of NF-kappaB activity in human prostate cancer cells is associated with suppression of angiogenesis, invasion and metastasis. Oncogene 20: 4188-4197, 2001.

16. Owens DM and Keyse SM: Differential regulation of MAP kinase signalling by dual-specificity protein phosphatases. Oncogene 26: 3203-3213, 2007.

17. Keyse SM: Dual-specificity MAP kinase phosphatases (MKPs) and cancer. Cancer Metastasis Rev 27: 253-261, 2008.

18. Arnoldussen YJ and Saatcioglu F: Dual specificity phosphatases in prostate cancer. Mol Cell Endocrinol 309: 1-7, 2009.

19. Xu S, Furukawa T, Kanai N, Sunamura M and Horii A: Abrogation of DUSP6 by hypermethylation in human pancreatic cancer. J Hum Genet 50: 159-167, 2005.

20. Furukawa T, Yatsuoka T, Youssef EM, et al: Genomic analysis of DUSP6, a dual specificity MAP kinase phosphatase, in pancreatic cancer. Cytogenet Cell Genet 82: 156-159, 1998.

21. Skrzypski M, Dziadziuszko R, Jassem E, et al: Main histologic types of non-small-cell lung cancer differ in expression of prognosis-related genes. Clin Lung Cancer 14: 666-673, 2013.

22. Piya S, Kim JY, Bae J, Seol DW, Moon AR and Kim TH: DUSP6 is a novel transcriptional target of p53 and regulates p53-mediated apoptosis by modulating expression levels of Bcl-2 family proteins. FEBS Lett 586: 4233-4240, 2012. 
23. Lee H, Kim JM, Huang SM, et al: Differential expression of DUSP6 with expression of ERK and Ki-67 in non-small cell lung carcinoma. Pathol Res Pract 207: 428-432, 2011.

24. Furukawa T, Sunamura M, Motoi F, Matsuno S and Horii A Potential tumor suppressive pathway involving DUSP6/MKP-3 in pancreatic cancer. Am J Pathol 162: 1807-1815, 2003.

25. Lim BJ, Jung SS, Choi SY and Lee CS: Expression of metastasis-associated molecules in non-small cell lung cancer and their prognostic significance. Mol Med Rep 3: 43-49, 2010.

26. Jin X, Yagi M, Akiyama N, et al: Matriptase activates stromelysin (MMP-3) and promotes tumor growth and angiogenesis. Cancer Sci 97: 1327-1334, 2006.

27. Liu HQ, Song S, Wang JH and Zhang SL: Expression of MMP-3 and TIMP-3 in gastric cancer tissue and its clinical significance. Oncol Lett 2: 1319-1322, 2011
28. Chen Y, Shi M, Yu GZ, et al: Interleukin-8, a promising predictor for prognosis of pancreatic cancer. World J Gastroenterol 18: 1123-1129, 2012.

29. Waugh DJ and Wilson C: The interleukin-8 pathway in cancer. Clin Cancer Res 14: 6735-6741, 2008.

30. Xie K: Interleukin-8 and human cancer biology. Cytokine Growth Factor Rev 12: 375-391, 2001.

31. Inoue K, Slaton JW, Eve BY, et al: Interleukin 8 expression regulates tumorigenicity and metastases in androgen-independent prostate cancer. Clin Cancer Res 6: 2104-2119, 2000.

32. Lee LF, Louie MC, Desai SJ, et al: Interleukin-8 confers androgen-independent growth and migration of LNCaP: differential effects of tyrosine kinases Src and FAK. Oncogene 23: 2197-2205, 2004 